\title{
MATLAB modeling to evaluate the ratio of packed cell volume
}

\author{
Zena Ahmed Alwan, Suhair Mohammed Yaseen, Huda Farooq Jameel \\ Department of Medical Instrumentation Techniques Engineering, Electrical Engineering Technical \\ College, Middle Technical University, Iraq
}

\begin{tabular}{l}
\hline \hline Article Info \\
\hline Article history: \\
Received Jan 14, 2021 \\
Revised Jun 8, 2021 \\
Accepted Jun 18, 2021 \\
\hline
\end{tabular}

Keywords:

Edge detected

Hematocrit

Packed cell volume

Red blood cells

\begin{abstract}
There are many direct and indirect methods of measuring the volumetric ratio of red blood cells relative to sample size and total blood, but the manual carcinogenic method remains the most stable and least expensive method despite the possibility of human error. The ratio percent of the packed red blood cell volume to the total volume of the blood sample is known as the packed cell volume (PCV). Our efforts were directed at finding a precise and economic method for PCV determination. Thereby, we designed a simple device consisting of a digital camera that is connected to a computer which uses a specific MATLAB program to process the blood images. As a result, the image can be read and the PCV can be calculated using a special code system. This new measuring system provides the capabilities and advantages to frequently comment on measured PCV with normal or abnormal, in comparison with normal values of PCV, which are stored in the computer. Our measurement method provides accurate results compared to the ruler results to alleviate the effort of clearing the hepatitis $\mathrm{C}$ virus from the blood (PCV). This system enables a swift and simple sample analysis and PCV identification.
\end{abstract}

This is an open access article under the CC BY-SA license.

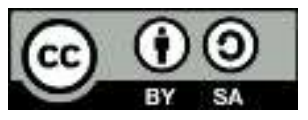

\section{Corresponding Author:}

Zena Ahmed Alwan

Department of Medical Instrumentation Techniques Engineering

Electrical Engineering Technical College

Middle Technical University, Iraq

Email: zena@mtu.edu.iq; zena782017@gmail.com

\section{INTRODUCTION}

Hemoglobin concentration and oxygen saturation are considered as an indication of a patient's ability to transport oxygen, these are routinely monitored or tested in any instance where oxygen transport is thought to be compromised. Additionally, hematocrit and total hemoglobin concentration are used preoperatively to monitor patients during procedures with a high risk of blood loss or hemorrhage. The measurements are also used in guiding clinical decisions to treat low blood volume, or anemia, through medication or blood transfusion [1]-[5]. The manual ruler method, used to estimate the volume of pressed blood cells, is one of the most used and least expensive methods and is therefore widely distributed in laboratories. A blood sample is taken from the patient using a capillary tube with anti-clotting material and ceterifuged.

The process of centrifuging the blood leads to the separation of the blood model into the aforementioned components, as shown in Figure 1. It is calculated within the size range of the red blood cells because this causes a measurement error. PCV is the volumetric percentage of erythrocytes to the size of the total blood model. 


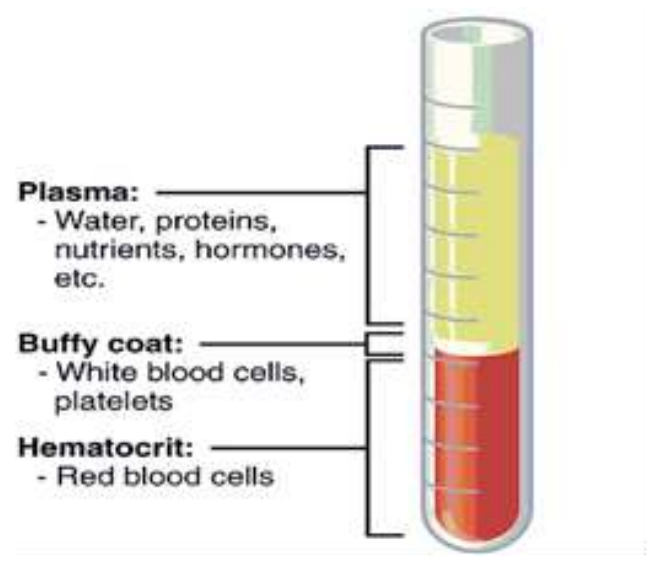

Figure 1. Model of centrifuged blood sample

The volumes are extracted by the following calculations:

$\mathrm{PCV}=\left(\frac{\text { High red blood cells in the form }}{\text { Total height of the blood model }}\right) \times 100$

PCV assessment was and remains a critical indicator for the diagnosis of many diseases and is one of the main laboratory analyses. The normal volumetric ratio of PCV is $45 \%$ for males and $40 \%$ for females [6]-[10]. One of the oldest and simplest methods and among most widely used for patients of different ages is the microhematocrit method as it requires a very small amount of blood, not exceeding one drop, and because it is easy to accomplish and very economic. However, more time is needed to separate the model and conduct the measurements, and there is the possibility of the overlapping of separate layers of plasma (trapped) if the analysis time exceeds ten minutes. This is in addition to the dependence of the measurement on manual reading and the associated probability of human error and the error of non-electronic manual devices.

Provided different classifiers to detect microcytic hypochromia using CBC and blood film features derived from a convolution neural network. The authors were used various evaluation metrics to achieved results (linear discriminant analysis (LDA), k-nearest neighbors (k-NN), support vector machine (SVM), and neural network classification principal component analysis (PCA)). However, the limited number of patients reduces the system complexity and researcher's time for getting data from different hospital to train the network. Also, the limited number of training with a large number of features cannot because it leads to overfitting [11]. Proposel of using a smartphone-obtained snapshot to extrapolate canine PCV and integrated into a mobile application with low-resource environments. The cumulative error of the calculation, on the other hand, may have been measured in comparison to the permissible error for the system. The findings of this research indicate that smartphone-obtained photographs may be used to estimate canine PCV. Unfortunately, this could not be measured because each sample had only been tested once. This was one of the study's limitations [12]. suggested a common operating procedure for calculating packed cell volume to reduce interoperator variability and improve accuracy. In a veterinary teaching hospital setting, the authors suggested a standard operating procedure (SOP) for canine PCV calculation that decreases operatordependent variance and increases accuracy. However, the number of samples was relatively limited, especially in the clinical staff classes, but this was due to participant availability; preferably, a greater number of participants would be recruited in related future experiments [13].

Design and implementation of electroic circuit which depended on Arduino board were performed by H. Farooq and S. yaseen to estimate PCV. The authors were calculated the PCV and Hb (hemoglobin) values, which makes analysis of PCV blood reliable and simple treatment. The system composed of Arduino board, red light (600nm), photocell, and LCD. However, the system has limited samples to analysis [14].

During measuring process of canine PCV, a method for identifying inters-and intra-operator variability, as well as the stage of errors was proposed by [15]. To assess the PCV packed cell volumes of three samples, the authors put undergraduate veterinary students and veterinary surgeons to the test. The information was gathered from 44 students and 11 veterinarians. PCV measurement, on the other hand, is highly helpful if the decisions taken to handle the cases of study are reliable and precise. Operator variance is a major aspect that must be overcome by adequate preparation and adherence to standard operating procedures. 
To overcome obstacles of measuring system complexity, limitation of samples, and error of light reflection, our suggested method is based mainly on taking a high resolution digital image of the blood model after centrifuging, then processing this image by MATLAB program to obtain the results of the measurement digitallyand comparision these results with stored normal values of PCV to introduce resulted PCV values with indication that value is normal or not.

\section{PROPOSED METHOD}

Although the manual ruler is the simplest and least expensive method-and is therefore widely used in laboratories-the absence of subdivisions within the ruler gradients and the fact that the reading depends on the correctness of the view of the analyst mean that there is a high probability of human error [16]-[18], resulting in an error percentage. Hence, we are looking for a more accurate way to calculate PCV. The new basic idea, which is the basis of the invention, is to calculate the value of PCV electronically.

The first step: After the process of separating the blood sample, the capillary tube is placed in the incision specified for it as the whole device is shown in Figure 2. The second step: the camera is turned on to take the required images for image processing and to show the results according to the patient's condition. The third step: the program will be launched and the images that were previously captured will be opened and they are processed according to the program placed in the coding system (code). The last step: It can show the results according to the context shown in Figure 3.

This idea is based on the design of a special graphical user interface used to calculate those ratios based on a set of commands that read the image. Then, the ratios are shown and modified using certain algorithms to produce a ready-to-read image, extracting the results with special equations within the code system. Measurement of PCV is performed by the following suggested system which consists of the parts below:

\subsection{Mechanical part}

As shown in Figure 2, this consists of the following components:

- Slot for the camera mode: Through this, the camera is placed in a certain way, according to the design, to take pictures of the blood sample that is in the capillary tube, then, the images are transmitted through a special cable from the camera to the computer for image processing according to the program prepared in advance.

- Incision of the capillary tube: This was designed according to the standard size of the capillary tube used for the analysis of PCV, it is placed after it has been introduced to the central separation device to induce separation between the red blood cells and the plasma to take the pictures and show the desired results.

- Plastic holder: This is designed according to the form to fit the requirements of the work and sensitivity in the analysis to get the best results.

- Base model: This has been added for the purpose of fixing the model and the accompanying movement of the device at work and during the accompanying difficulties, especially in analysis laboratories.

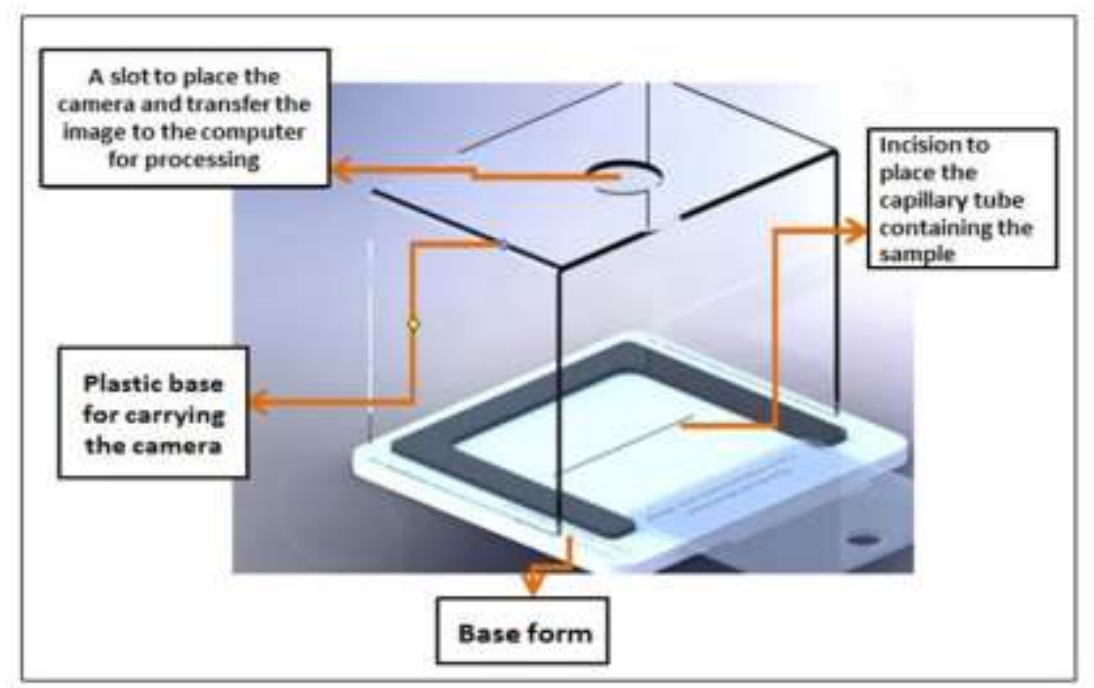

Figure 2. The mechanical part 


\subsection{Electrical part}

Computer: This is the controlling part of the system, achieved using the MATLAB program design master program, which controls the image processing and indicates the required measurements through the image of the capillary tube containing the blood sample of the person to be tested. Data transmission cable: This is used to transfer data through the connection between the computer and the camera. Digital camera: This is an electronic machine that takes photographs and stores them electronically instead of using film like conventional cameras. Modern digital cameras have become so versatile that some can record audio or video using a memory card, which varies in size to enable it to store more images. The camera is used to take a picture of the separated blood sample and the image is transmitted via a special cable to the computer to be processed by a special code to extract the value for the number of blood cells. The blood sample and camera specifications used in this device are effectively 7.2 megapixels with a high sensitivity of ISO 1000 and approximately 380 frames.

\subsection{Programmatic part}

MATLAB (Matrix-Laboratory) is a leading program in mathematical engineering and mathematical applications. MATLAB has the ability to compute matrices, graph mathematical functions, implement various algorithms, create graphical user interfaces, and communicate with programs written in other languages, including $\mathrm{C}-, \mathrm{C}++$, Java, and Fortran. This program is also used to draw two-dimensional graphs and to solve difficult mathematical equations and digital image processing to show the best results [19]-[22].

Here, we explain the interface developed to conduct the analysis of the image and to show the desired results. As shown in Figure 3, the interface includes the following parts:

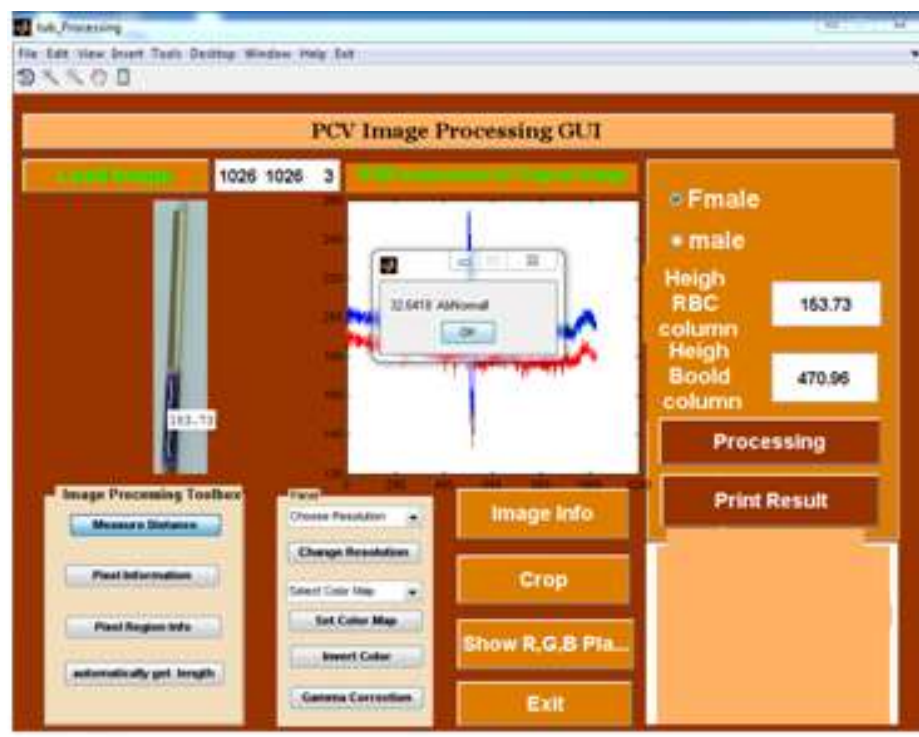

Figure 3. The interface of the program

- Download the image: Download an image from the blood sample to the computer to be processed to achieve the result; the image is in the following forms: JPG, BMP, JPEG, PNG.

- Axis (G1): The place where the image from the computer is processed using the image processing code graphical user interface in the MATLAB program.

- Distance measurement: A programmed ruler is used to calculate the total height of a blood column in a unit of measurement.

- Pixel information: This displays the information for each pixel in the image, whereby it is coordinated in the form of matrices (Pixel info (X, Y) [R G B]).

- Pixel area information: Displays all the image pixels in a new list.

- Get the length automatically: The length of the model of the whole blood column and the length of the column of red blood cells (RBC) are taken automatically.

- Image information: This command displays special information that is processed, such as file name, file status data, image format, image width, and image length.

- Cutting: This command is used to cut the image and extract the part to be processed. 
- RGB level display: This command displays the primary colors in the image matrix (red level, green level, blue level).

- Radio button: This part is used to choose the sex of the patient (male and female) to distinguish the value of compressed blood cells depending on the sex of the patient.

- Height of the red blood cell column: This part is used to write the value of the height of the red blood cell column.

- Height of the blood column pillar: Measurement of the height of the pillar of the whole blood model, which includes plasma, white blood cells, and red blood cells.

- Treatment: This is the most important part of the program and calculates the value of the pressed blood cells using the law of the ratio between the height of the red blood cell column to the height of the whole blood column according to the following formula:

$\mathrm{PCV}=\left(h /{ }_{H}\right) \times 100$

where:

$\mathrm{h}=$ the height of the compressed erythrocyte column.

$\mathrm{H}=$ full blood column height

- The result of printing: This is where the result of the analysis is printed.

- Exit: This section is used to close the program.

\section{RESULTS AND DISSCUSION}

After the implementation of the PCV measuring device, the program is run using the MATLAB software R2017b [23]-[26], which is considered a very high-level language because it deals with commands that are implemented by any program. The program is run according to the following steps:

- After the process of the separation of the blood model, as shown in the abovementioned practical steps, a capillary tube is placed in the joint in the specified incision, as shown in the device overview in Figure 2.

- The camera is turned on to take the pictures required for image processing and to show the results according to the patient's condition.

- The program is run, opening the images taken above and processing them according to the program set in the code system of the image processing.

- We can show the results according to the context shown in Tables 1 and 2. Which illustrate the difference between the PCV values calculated using image processing and the microhematicrite ruler. As shown in the table, reading with a microhematicrite ruler depends on the accuracy and craftsmanship of the examiner. It is clearify that the process the images are esay because the image is very high precision and has only two dimensions, and the reading is more stable. That usefule for medical analyses in diagnose the disease.

Table 1. Sex: female 1

\begin{tabular}{cccl}
\hline No. of tests & Read using microhematicrite ruler & Read using image processing & Error ratio \\
\hline 1 & 34 & 32.3029 & 2.329 \\
2 & 32 & 32.3251 & 0.4251 \\
3 & 33 & 32.3418 & 1.3418 \\
\hline
\end{tabular}

Table 2. Sex: female 2

\begin{tabular}{cccc}
\hline No. of tests & Read using microhematicrite ruler & Read using image processing & Error ratio \\
\hline 1 & 38 & 38.0134 & 0.0134 \\
2 & 37 & 38.0131 & 1.0131 \\
3 & 39 & 38.0251 & 1.0251 \\
\hline
\end{tabular}

\section{CONCLUSIONS}

The value of PCV is measured electronically, which is the current fundamental that paper is based on. The aim is to create a specific graphical user interface to quantify these ratios using a series of commands for processing, viewing, and manipulating the image with algorithms to generate a ready-to-read image, and then extracting the result using special equations inside the coding system. According to the experiment involving the abovementioned tests, the following can be concluded that reduction of human errors and increased accuracy in the results obtained. Table 1 illustrates this. Rapid completion of the analysis. It is 
possible to analyze a large number of models at once numbered and takes a group photo. The ability to analyze samples remotely as soon as they are available. A preliminary judgment on the results of the analysis of the models, whether they are natural or unnatural, whereby the natural ratios were stored in the program and the appearance of the result is written on the program interface. The device works on a regular battery that can be recharged, meaning it does not need an electrical source or generator in the system. It is considered a racing model to measure the volume ratio of red blood cells using image processing. Subsequently, we suggest that this new device be used to measure blood samples and find PCV values for blood models in hospitals and analytical laboratories in place of the old methods, in which the error rate is questionable. In addition, we can take advantage of developments in the field of electronics and computers.

\section{FUTURE WORK}

Future work could design and implement a device for measuring the volume of compressed red blood cells can be used multiple samples in the same time. Also, could connect the camera by internet that lets a database for all Analysts to use it and connect the computer with camera by wifi to allow to use one computer device for all laboratories.

\section{REFERENCES}

[1] P. C. Hebert et al. "A multicenter, randomized, controlled clinical trial of transfusion requirements in critical care," The New England Journal of Medicine, vol. 340, no. 6, pp. 409-417, 1999, doi: 10.1056/NEJM199902113400601.

[2] J. S. Ruckman, "A comparative study of total hemoglobin measurement technology: noninvasive pulse co-oximetry and conventional methods", M.S. thesis, University of Connecticut, USA, 2011.

[3] M. Wattana, B. Siriluk, and S. Khotwit, "Counting and Separating Damaged Seeds of Soybean Seeds using Image Processing," International Journal on Advanced Science, Engineering and Information Technology, vol. 8, no. 4, pp. 1366-1371, 2018.

[4] B. Yang, C. Liu, W. Zheng, and S. Liu, "Motion prediction via online instantaneous frequency estimation for vision-based beating heart tracking," Information Fusion, vol. 35, pp. 58-67, 2017, doi: 10.1016/j.inffus.2016.09.004.

[5] A. A. Naser, T. Hassan, and M. Naji Majeed, "Detection of hemoglobinophathies in hypochromic, microcytic and sickeled cell blood films by hemoglobin electrophoresis," Thi-Qar Medical Journal (TQMJ), vol. 5, no.1, 2011, pp.139-148.

[6] L. Wheeler, "Chapter 11: Clinical Laboratory Instrumentation," in Medical Instrumentation: Application and Design, J. G. Webster, A. J. Nimunkar, 4th ed. Hoboken, New York, USA: John Wiley \& Sons, 2010, pp. 498-527.

[7] B. S. Bull, J. A. Koepka, E. Simson, and O. W. van Assendelft, "Procedure for Determining Packed Cell Volume by the Microhematocrit Method; Approved Standard-Third Edition," Carden Jennings Publishing Co., Ltd. Third Edition, Laboratory Hematology 7, vol. 20, no. 18, pp. 191-196, 2001.

[8] K. Sembulingam and P. Sembulingam, Assentials Of Medical Physiology, Chapter 7, Ed. $6^{\text {th }}$, India: Jaypee Brothers Medical Publisher (P) Ltd, 2010, pp. 59-88.

[9] B. H. Estridge, A. P. Reynolds and N. J.Walters, "Basic Medical Laboratory Techniques," Delmar Thomson Learning, Ed. $4^{\text {th }}$, unit 2, Albany, New York: Thomson Learning, 2000, pp. 125-127.

[10] L. Lamhaut, "Comparison Between a New Noninvasive Continuous Technology of Spectrophotometry-based and RBC Count for Hemoglobin Monitoring During Surgery with Hemorrhagic Risk," European Journal of Anaesthesiology, vol. 27, pp. 60-61, 2010, doi: 10.1097/00003643-201006121-00192.

[11] C. Breheny, A. Brown, I. Handel, and A. G. Gow, "Inter- and intra-operator variability in the analysis of packed cell volume," Journal of Small Animal Practice, vol. 58, no. 1, pp. 29-34, 2016, doi: 10.1111/jsap.12603.

[12] S. Purwar, R. K. Tripathi, R. Ranjan, and R. Saxena, "Detection of microcytic hypochromia using cbc and blood film features extracted from convolution neural network by different classifiers," Multimedia Tools and Applications, vol. 79, pp. 4573-4595, 2020, doi: 10.1007/s11042-019-07927-0.

[13] C. R. Breheny, S. E. Kinsey, and A. G. Gow, "The use of smartphone obtained images to extrapolate canine packed cell volume," Journal of Veterinary Emergency and Critical Care, vol. 30, no. 4, pp. 426-435, 2020, doi: 10.1111/vec.12961

[14] C. R. Breheny, J. P. A. Salgado, N. X. Bommer, I. Handel, and A. G. Gow, "Standard operating procedure reduces interoperator variation and improves accuracy when measuring packed cell volume," Veterinary Record, vol. 184, no. 9, pp. 283-283, 2019, doi: 10.1136/vr.104774.

[15] H. F. J. Baban and S. M. H. Yaseen, "Design and Implementation of PCV Device Based on Arduino Board," Journal of Al-Nahrain University, vol. 19, no. 4, pp. 16-20, 2016.

[16] M. Evans, Y. He, L. Maglaras, and H. Janicke, "HEART-IS: A novel technique for evaluating human error-related information security incidents," Computers \& Security, vol. 80, pp. 74-89, 2019, doi: 10.1016/j.cose.2018.09.002.

[17] S. D. Bolboacă, "Medical diagnostic tests: a review of test anatomy, phases, and statistical treatment of data," Computational and mathematical methods in medicine, vol. 2019, 2019, doi: 10.1155/2019/1891569.

[18] C. M. U. Chikere, K. Wilson, S. Graziadio, L. Vale and A. J. Allen, "Diagnostic test evaluation methodology: A systematic review of methods employed to evaluate diagnostic tests in the absence of gold standard-An update," PLoS One, vol. 14, no. 10, p. e0223832, 2019, doi: 10.1371/journal.pone.0223832. 
[19] J. Gibson and A. Bovik, "Handbook of Image and Video Processing," in Communications, Networking and Multimedia, Ed. $2^{\text {nd }}$, USA: CRC Press, 2005.

[20] Z. Rustam, A. Kamalia, R. Hidayat, F. Subroto, and S. A. Suryansyah "Comparison of Fuzzy C-Means, Fuzzy Kernel C-Means, and Fuzzy Kernel Robust C-Means to Classify Thalassemia Data," International Journal on Advanced Science, Engineering and Information Technology, vol. 9, no. 4, pp. 1205-1210, 2019.

[21] A. Lotfy, W. Anis, and J. V. M. Halim, "Design PV system for a small GEO satellite and studying the effect of using different types of propulsion," International Journal of Advances in Applied Sciences (IJAAS), vol. 8, no. 1, pp. 54-63, 2019, doi: 10.11591/ijaas.v8.i1.pp54-63.

[22] S. Bachu, S. Choubey, and A. Choubey, "Noval advance non-linear descriptor and characteristic equivalency to predict CT from MRI image," International Journal of Informatics and Communication Technology (IJ-ICT), vol. 9, no. 1, pp. 46-56, 2020, doi: 10.11591/ijict.v9i1.pp46-56.

[23] M. Nixon and A. S. Aguado, Feature Extraction \& Image Processing for Computer Vision (Third Edition), USA: Academic press, 2019, doi: 10.1016/B978-0-12-396549-3.00010-0.

[24] T. G. Devi and P. Neelamegam, "Image processing based rice plant leaves diseases in Thanjavur, Tamilnadu," Cluster Computing, vol. 22, pp. 13415-13428, 2019, doi: 10.1007/s10586-018-1949-x.

[25] S. Papadopoulos et al., "Image clustering through community detection on hybrid image similarity graphs," 2010 IEEE International Conference on Image Processing, 2010, pp. 2353-2356, doi: 10.1109/ICIP.2010.5653478.

[26] J.-D. Tournier et al., "MRtrix3: A fast, flexible and open software framework for medical image processing and visualisation," NeuroImage, vol. 202, 2019, doi: 10.1016/j.neuroimage.2019.116137.

\section{BIOGRAPHIES OF AUTHORS}

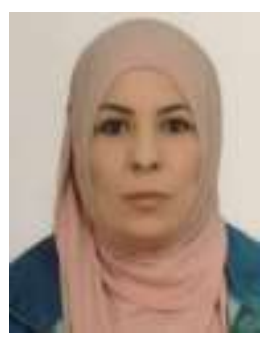

Zena Ahmed Alwan has gotten her Bachelor certificate in Computer Sciences from the University of Baghdad in 2002. With a similar University She got her Master certificate in the Computer Sciences in 2005. She is at present a lecturer at the Department of Medical Instrumentation Techniques Engineering in Electrical Engineering Technical College in the Middle Technical University, Iraq, her exploration interest Digital Image Processing, Information Hiding and Pattern Recognition.

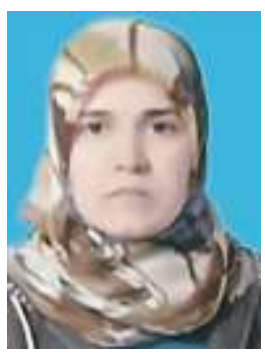

Suhair Mohammed Yaseen received her B.Sc. in Chemistry Sciences from the University of Baghdad. She received her M.Sc. degree in the Chemistry Sciences from the same University and now she Ph.D. student at last stage in field of Anatytical Chemistry because of her work in college of Electrical Engineering Techniques, she interesting in Chemistry Application Medical Engineering.

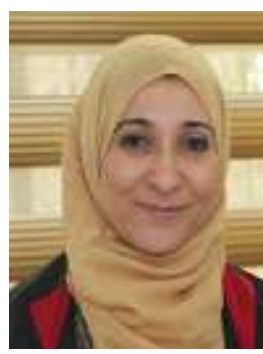

Huda Farooq Jameel from Middle Technical University/Electrical Engineering Technical College/Medical Instrumentation Techniques Engineering Department. She received her Master degree in Electronic Medical Instrumentation Techniques Engineering in 2020. She interesting in this field and in digital image processing. 